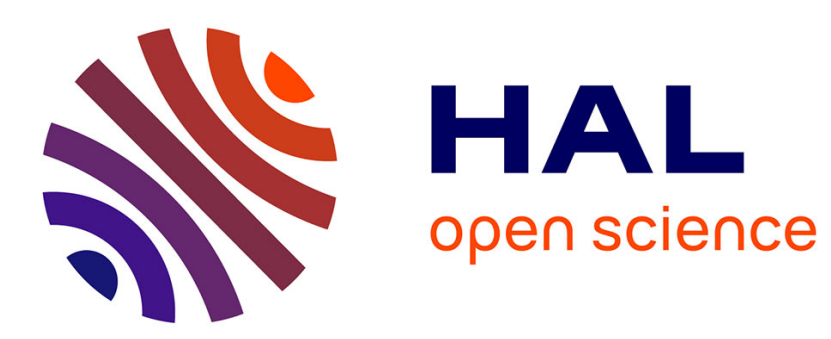

\title{
Measuring the Stray Capacitance of Solenoids with a Transmitting and a Receiving Coil
}

\author{
Andrea Mariscotti
}

\section{To cite this version:}

Andrea Mariscotti. Measuring the Stray Capacitance of Solenoids with a Transmitting and a Receiving Coil. MTA Review / Military Technical Academy Review, 2011, 18 (1), pp.47-56. 10.2478/v10178011-0005-4 . hal-02883572

\section{HAL Id: hal-02883572 \\ https://hal.science/hal-02883572}

Submitted on 29 Jun 2020

HAL is a multi-disciplinary open access archive for the deposit and dissemination of scientific research documents, whether they are published or not. The documents may come from teaching and research institutions in France or abroad, or from public or private research centers.
L'archive ouverte pluridisciplinaire HAL, est destinée au dépôt et à la diffusion de documents scientifiques de niveau recherche, publiés ou non, émanant des établissements d'enseignement et de recherche français ou étrangers, des laboratoires publics ou privés. 


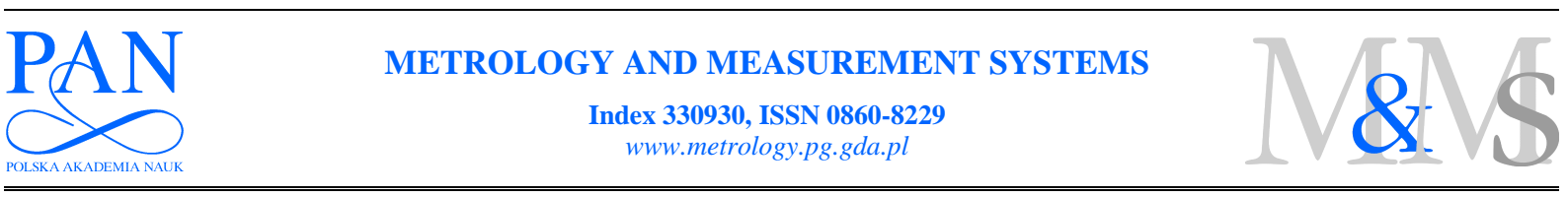

\title{
MEASURING THE STRAY CAPACITANCE OF SOLENOIDS WITH A TRANSMITTING AND A RECEIVING COIL
}

\author{
A. Mariscotti \\ Università di Genova, DINAEL, Via all'Opera Pia, 11 - 16145 Genova, (凹andrea.mariscotti@ unige.it, +390103532169, \\ $+390103532700)$
}

\begin{abstract}
Air core solenoids, possibly single layer and with significant spacing between turns, are often used to ensure low stray capacitance, as they are used as part of many sensors and instruments. The problem of the correct estimation of the stray capacitance is relevant both during design and to validate measurement results; the expected value is so low to be influenced by any stray capacitance of the external measurement instrument. A simplified method is proposed that does not perturb the stray capacitance of the solenoid under test; the method is based on resonance with an external capacitor and on the use of a linear regression technique.
\end{abstract}

Keywords: Capacitance measurement, Uncertainty, Stray capacitance, Linear regression, Resonance condition

(C) 2011 Polish Academy of Sciences. All rights reserved

\section{Introduction}

The present work originated from the need of determining the stray capacitance terms of a high voltage Rogowski coil with a wide frequency range [1],[2]. This solenoid was designed for high voltage immunity by selecting a coaxial conductor instead of a single conductor and the stray capacitance is represented by an articulated scheme of capacitance terms between adjacent and non-adjacent turns. This design is different from the more usual solenoid with an external cylindrical shield, that features a preponderant winding-to-shield (or self-) capacitance term, that reduces the relevance of the said turn-to-turn (or mutual) capacitance terms. The capacitance terms and their distribution along the solenoid are relevant for two reasons: they determine the overall frequency response of the solenoid and how an external electric field couples to the solenoid elements, thus determining its susceptibility.

Other instruments that use solenoids of various shapes and thus need an accurate determination of the stray capacitance, at least for the characterization of the frequency response at the upper extreme of the frequency interval, are a magnetic field probe with triaxial windings operating up to $1 \mathrm{MHz}$ [3] and a non-invasive rail current sensor [4]. In both cases the windings were built with an inter-turn gap of one wire diameter, chosen because of construction ease. From a thorough examination of the literature on the subject it was evident that there are no satisfactory closed form expressions for the calculation of the stray capacitance term of air solenoids with one layer and significant inter-turn gap.

\section{Stray capacitance measurement}

There are a few references where closed form expressions and some measurement results are presented [5]-[7], with limited agreement between the presented results. Moreover, the case of wide separation between adjacent turns is rarely treated accurately enough. For this reason the problem of the design of an experimental setup and a measurement procedure is 
considered here. In the following only the literature results that are judged trustable are reported for reference. Medhurst [6],[7] presented a semi-empirical formula, that fits the results of several measurements on different solenoids:

$$
C_{M e d 1}=D[11.26 l / D+8+27 \sqrt{D / l}],
$$

where $D[\mathrm{~m}]$ is the solenoid diameter and $l[\mathrm{~m}]$ is the solenoid length. The first term is derived from the Nagaoka formula for the solenoid inductance, the other two are determined on an empirical basis.

Another formula credited to Medhurst, but for which a precise bibliographic reference could not be found, is

$$
C_{\text {Med } 2}=\frac{4 \varepsilon_{0} l}{\pi}\left[0.71 D / l+1+2.4(D / l)^{1.5}\right] .
$$

The case considered in [5] regards inductors with an external shield and is not applicable to the present problem, as it was evident by inspection of the curves behavior, opposite to Medhurst findings and to what has been observed experimentally.

\section{Test setup and experimental results}

The test setup is made of a signal source, a coupling method to apply the signal to the solenoid under test (s.u.t.) and a measurement instrument to detect the resonance condition. The inductance of the s.u.t. is preliminarily determined by calculation in order to define the frequency range of interest and the prescribed values of the external resonating capacitor.

\subsection{Solenoid inductance}

Two air solenoids are considered with two very different wire diameters as reference examples in the following: solenoid A with $r_{1}=1.0 \mathrm{~mm}$ of enameled copper wire, and solenoid B with $r_{2}=4 \mathrm{~mm}$, using a thick wire, represented by the external shield of a RG58 coaxial cable. The number of turns $N$ is made variable between 10 and 40 turns.

The influence of the inductive part of the solenoid and the value of the natural self resonance frequency were evaluated first [8][10]. The application of the Webster-Havelock formula (including Rosa correction for turns separation), with wire diameter $r$ in $\mathrm{cm}$, turn pitch $p$ in $\mathrm{cm}$, solenoid radius $R$ in $\mathrm{cm}$, length $l$ in $\mathrm{cm}, N$ the number of turns)

$$
\begin{aligned}
& L_{W, H, R}=4 \pi^{2} \frac{R^{2} N^{2}}{l}\left[1-\frac{8}{3 \pi} \frac{R}{l}+\frac{1}{2} \frac{R^{2}}{l^{2}}-\frac{1}{4} \frac{R^{4}}{l^{4}}+\frac{5}{16} \frac{R^{6}}{l^{6}}-\frac{35}{64} \frac{R^{8}}{l^{8}}+\cdots\right]-4 \pi R N(A+B), \\
& A=3.4904 \ln (r / p) \quad B=0.25 \text { if } n<15,0.3 \text { if } n \geq 15
\end{aligned}
$$

gives a variable inductance of 2 to $6 \mu \mathrm{H}$ for $N$ ranging between 10 and 40 (as shown in Fig. 1), with the extreme configuration of $r=1 \mathrm{~mm}$ and $p=10 \mathrm{~mm}$ included only for completeness. 


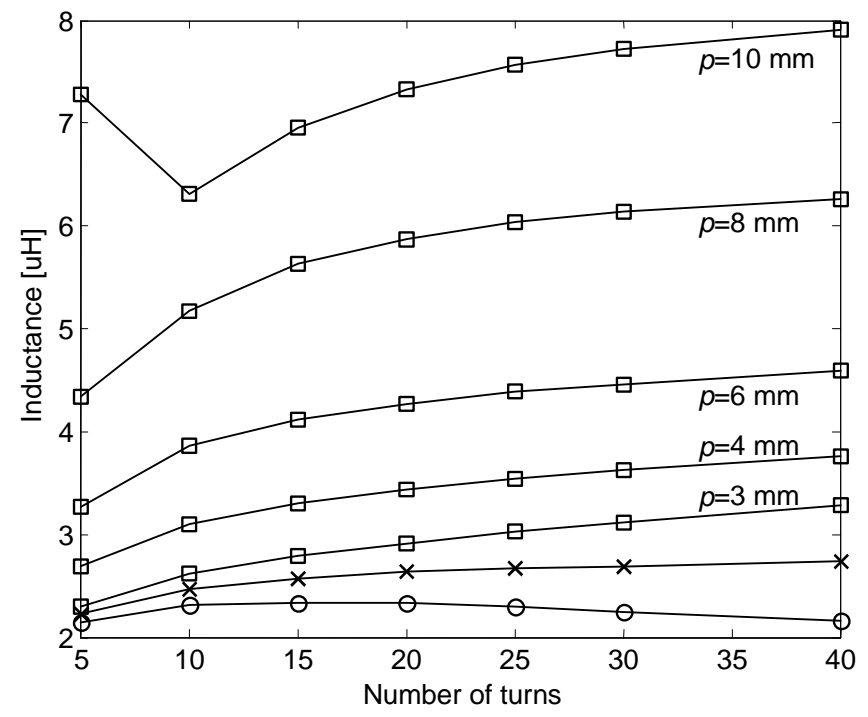

Fig. 1. Calculation of the solenoid inductance using (1): $r=1 \mathrm{~mm}$, variable pitch $p$ (square), $r=2 \mathrm{~mm} p=10 \mathrm{~mm}$ (cross), $\mathrm{r}=4 \mathrm{~mm} p=10 \mathrm{~mm}$ (circle)

The problem of the dependency of the s.u.t. inductance on frequency was considered. If the skin depth phenomenon is considered, the geometrical external inductance $L_{e x t}$ is constant since any change of the current distribution in the wire cross section is symmetrical with respect to the wire axis. The internal inductance $L_{\text {int }}$, on the contrary, may be estimated over the frequency interval of interest by the practical formulae appearing in [8] or by numerical computation (methods are summarized in [9]); for a single turn the ratio $L_{\text {int }} / L_{\text {ext }}$ is already less than $1 \%$ in all conditions, but if the total s.u.t. inductance is considered, then $L_{e x t}$ increases more rapidly than $L_{\text {int }}$ with the number of turns and the ratio is even smaller, less than $0.1 \%$ above ten turns.

With an a-priori estimated stray capacitance $C_{p}$ of few $\mathrm{pF}$, the self resonance frequency is then estimated between 20 and $80 \mathrm{MHz}$, so that a direct measurement of the stray capacitance by a LCR bridge is not possible, since the inductive reactance in parallel shunts the capacitive reactance under measurement over too an extended frequency range. So, the measurement of the stray capacitance was designed using two different techniques and the setups as described in [10]: in both cases the s.u.t. is connected to the signal source and to the measuring equipment with no galvanic coupling, by using either a transmitting coil and a clamp-on current probe on the s.u.t conductor, or twin transmitting and receiving coils coaxial with and on either sides of the s.u.t.

\subsection{Transmitting and receiving coils method}

The excitation signal is applied to the s.u.t. (solenoid under test) by means of a transmitting coil (TC) with a very small number of turns, so that its self resonance frequency is moved well above the frequency range of interest. The s.u.t. is connected to an external capacitor $C_{e x t}$ to drive its resonance on a suitable frequency interval (see subsection C). In [10] it was proposed to detect the resonance condition by either 1) a current probe in series to the coil (the output variable is the current circulating in the s.u.t., measured with a current probe featuring a negligible insertion impedance $(<1 \Omega)$ ) or 2$)$ a receiving coil, RX coupled to the s.u.t. and similar or identical to the TC, with the output variable being the RX coil voltage. The use of the current probe and method 1) doesn't ensure that the total resonating current is measured, since a fraction of it recloses to the stray capacitance (that establishes between each pair of turns), before reaching the terminals of the s.u.t.. In this way it is possible (or it cannot be excluded) that the current probe method gives a systematic error that biases the $C_{p}$ 
estimate. On the contrary, the use with method 2) of two identical TX and RX coils mounted coaxially on a supporting plastic pipe is able to measure the resonating circuit as a whole; the equivalent circuit is shown in Fig. 2. Since the method 1) is more practical it will be considered in a future development.

The frequency of the tracking generator is swept over the set frequency interval, by applying the voltage $V_{t x}$ to the TX coil through the RF amplifier (optional), and the received voltage $V_{r x}$ is read on the spectrum analyzer input channel. Both TX and RX coils are designed to have a very large self-resonance frequency and a small reactance, so that the use of a terminating $50 \Omega$ resistor ensures both impedance matching and linearization of the circulating current over a fairly wide frequency interval. For the RX coil, since the output voltage of the tracking generator $V_{t g}$ is specified for a $R=50 \Omega$ matching condition, for the frequency values $f$ at which $X_{t x}\left(=2 \pi f L_{t x}\right)<<R$, the circulating current $I_{t x}$ is given readily as $I_{t x}=G V_{t g} / 50$, where $G$ is the RF amplifier gain.

The RX coil receives a fraction of the field sourced by the TX coil related to the normal coupling between the TX and RX coils (due to their geometrical characteristics, such as diameter, number of turns, turn spacing, coil length and inter-coil distance). A second effect is the additional shunting due to the interposed s.u.t.; when the s.u.t. resonance occurs its impedance reaches a maximum, the shunting effect reaches a minimum and the received voltage $V_{r x}$ reaches a maximum.

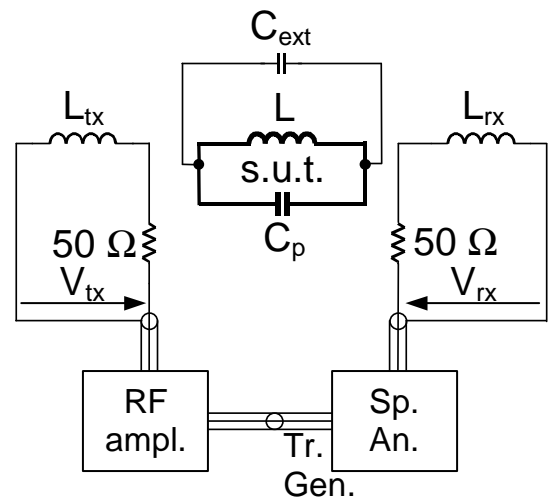

Fig. 2. Measurement setup with coupled transmitter and receiver coils and interposed s.u.t.

It is underlined that this method doesn't rely on absolute properties of the test setup, but rather on the change in the received voltage due to the insertion of the s.u.t.. When this operation is performed care must be given that the positioning is accurate to ensure repeatability also of intermediate results, such as $V_{r x}$, and doesn't modify accidentally the rest of the test setup.

To demonstrate the characteristics of the measurement setup, two TX and RX coils have been tested for their behavior at radiofrequency. Since an external capacitor may be used, the attention here is on a frequency interval that is extended from the $[20,80] \mathrm{MHz}$ to lower frequency down to approximately a few MHz; the TX and RX coils are winded on a plastic pipe that keeps them coaxial and allows to fix the turn-to-turn separation (the turn pitch) by applying adhesive tape. The aspects that have been considered are:

- the input impedance with respect to the reference value of $50 \Omega$, expressed in terms of the reflection coefficient and measured by means of a 1 port reflection loss test;

- the transfer function between the TX and the RX coil, measured by means of a 2 port test;

- the sensitivity of the signal to noise ratio and the resonance frequency to the longitudinal position of the s.u.t. between the two extreme points at the TX and RX coils.

The results of the characterization of the measurement setup are shown in Fig. 3 and 4. 

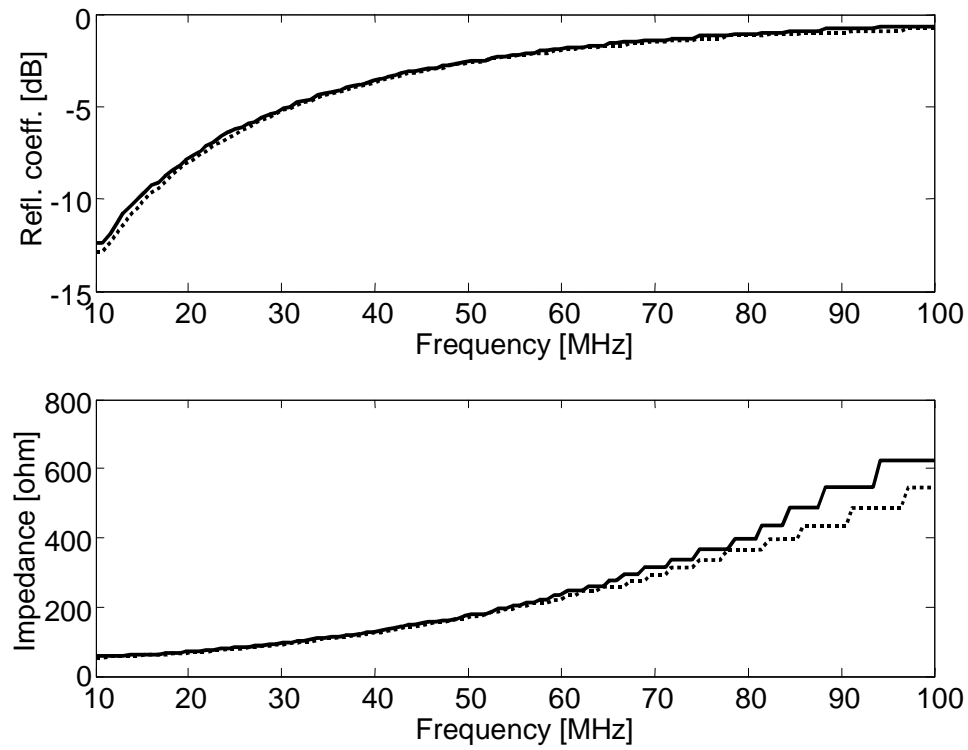

Fig. 3. Reflection coefficient and input impedance of the TX (solid) and RX (dotted) coils

The transfer function $H(f)$ was measured for variable distance $d$ between TX and RX coils, with the distance measured between the mid point of each coil.

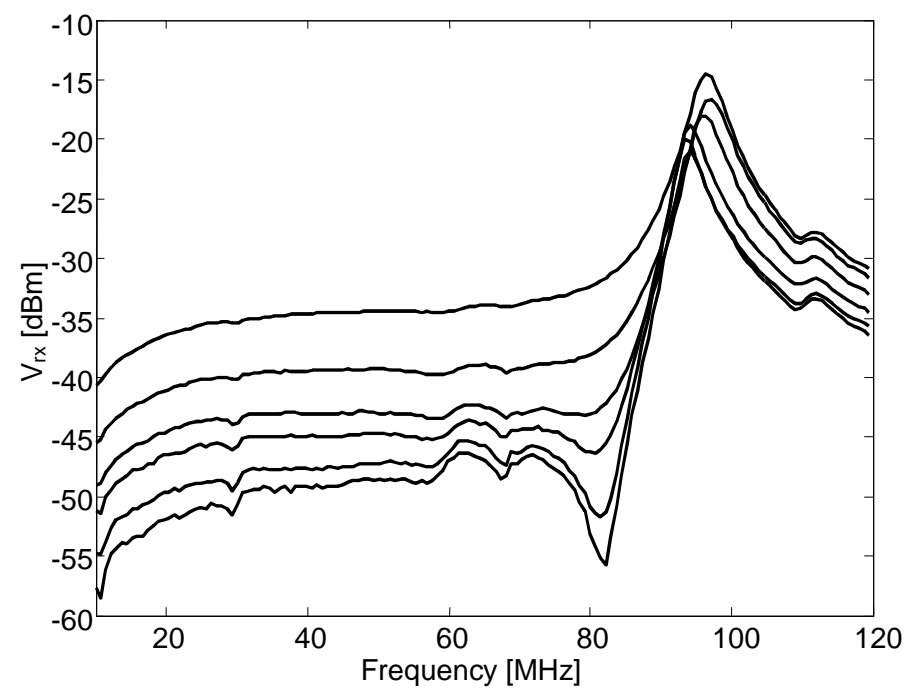

Fig. 4. $H(f)$ versus distance $d=D, 1.25 D, 1.5 D, 1.75 D, 2 D, 2.5 D$, with the coil diameter $D=4 \mathrm{~cm}$

The curves shown in Fig. 4 for increasing distance show a good uniformity and the first two, for $d=D$ and $1.25 D$, are almost free of any resonance behavior.

It is remembered that since the proposed method is based on a relative measurement the input impedance and the transfer function don't influence the final result, as long as they keep within an acceptable range, that is a reflection coefficient compatible with the operation of the $\mathrm{RF}$ amplifier and the absence of relevant peaks in the transfer function that could be confused with the correct one.

To this aim the sensitivity of the resonance peak in $V_{r x}$ with respect to the positioning of s.u.t. between the TX and RX coils is investigated, in terms of amplitude and frequency value. The s.u.t. is made of seven turns of thin wire on a $2.5 \mathrm{~cm}$ coil former and enters the test setup from the TX coil side, moving towards the RX coil by $5 \mathrm{~mm}$ at a time ( $4 \mathrm{~mm}$ in the central zone); the TX and RX coils are located at $d=D=4 \mathrm{~cm}$ distance. 


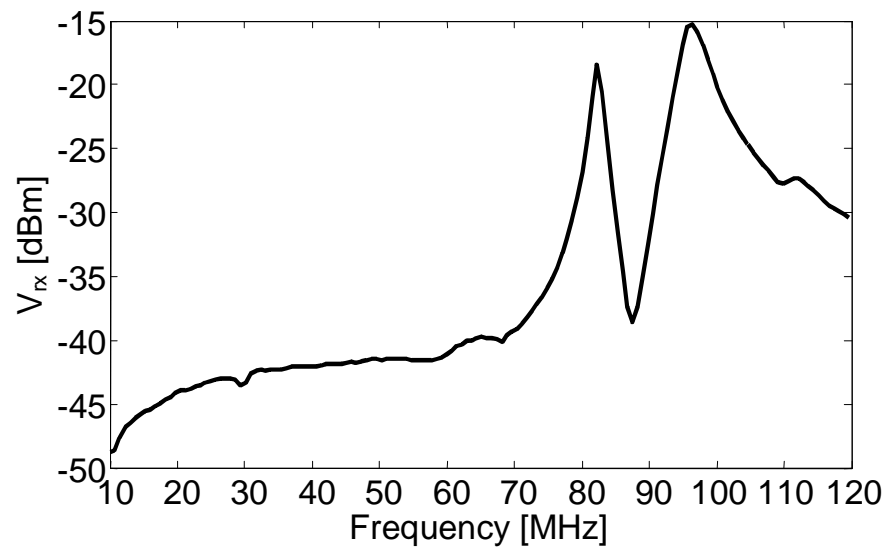

Fig. 5. Example of resonance peak

Table 1. Amplitude and frequency of the resonance peak versus s.u.t. position

\begin{tabular}{|c|c|c|}
\hline s.u.t. position $x[\mathrm{~cm}]$ & Amplitude $[\mathrm{dB}]$ & $\begin{array}{c}\text { Frequency } \\
{[\mathrm{MHz}]}\end{array}$ \\
\hline 0 (@ TX) & -28.7 & 86.745 \\
\hline 0.5 & -25.5 & 85.255 \\
\hline 1.0 & -21.4 & 83.166 \\
\hline 1.4 & -18.5 & 82.410 \\
\hline 1.8 & -18.1 & 82.293 \\
\hline 2.2 & -18.4 & 82.302 \\
\hline 2.6 & -21.1 & 82.278 \\
\hline 3.0 & -22.1 & 83.283 \\
\hline 3.5 & -29.8 & 85.231 \\
\hline $4.0(@ \mathrm{RX})$ & -31.8 & 85.514 \\
\hline
\end{tabular}

In Fig. 5 the resonance peak on the left is the correct peak, while the peak on the right is due to a resonance of the present test setup arrangement and is at the extreme of the considered frequency interval. In the reported example the s.u.t. is made resonating on its own parasitic capacitance; to bring the self resonance in the desired frequency interval and to apply the method of the linear regression considered in the next subsection an external capacitor is connected at the terminals of the s.u.t..

The values reported in Table 1 show that in the central portion of the setup the change of the resonance frequency for small positioning errors is limited to about $6 \mathrm{ppm} / \mathrm{mm}$.

\subsection{Use of external capacitor}

For the measurement setup described in the previous subsection, since the self resonance of the solenoid may be scarcely visible and/or positioned at too a high frequency, a linear regression technique is followed [7], using external resonating capacitors. The resonance frequency is determined by the joint effect of the total equivalent parasitic capacitance $C_{p}$ and the external capacitor $C_{\text {ext }}$ :

$$
f_{r}=\frac{1}{2 \pi \sqrt{L\left(C_{p}+C_{e x t}\right)}} .
$$

It is convenient to express the external capacitor as a linear function of the square of the resonance wavelength with the unknown stray capacitance $C_{p}$ as the intercept: 


$$
C_{e x t, i}=\frac{1}{4 \pi^{2} c^{2} L} \lambda_{i}^{2}-C_{p}=A \lambda_{i}^{2}-C_{p},
$$

where $c$ is the speed of light and the pairs $\left(\lambda_{i}^{2}, C_{e x t, i}\right)$ indicate the measurement points.

Several resonating capacitors $C_{e x t}$ were connected to the s.u.t. output terminals and the resonance frequency values recorded. The adopted Least Mean Square algorithm for the determination of the linear regression coefficients (slope $A$ and intercept $-C_{p}$ ) is sensitive to the location of the reference points (see Section 4 for details), so the $C_{e x t}$ values were selected clustered at the two extremes.

\subsection{Sample measurement results}

The tested case corresponds to one solenoid made of thick hollow cylindrical wire (the external shield of a RG58 coaxial cable, with a radius of $4 \mathrm{~mm}$ ) with a progressively smaller number of turns $(N=10,15,20,25,30,40)$, the cable in excess being cut away [10]. The measurements are made with eight $C_{\text {ext }}$ values between 27 and $30 \mathrm{pF}$ and eight $C_{\text {ext }}$ values between 2.17 and $2.21 \mathrm{nF}$, for a total $n=16$ measurements.

The results of $C_{p}$ determination are shown in Fig. 6 together with the estimated uncertainty intervals evaluated as described in the next Section.

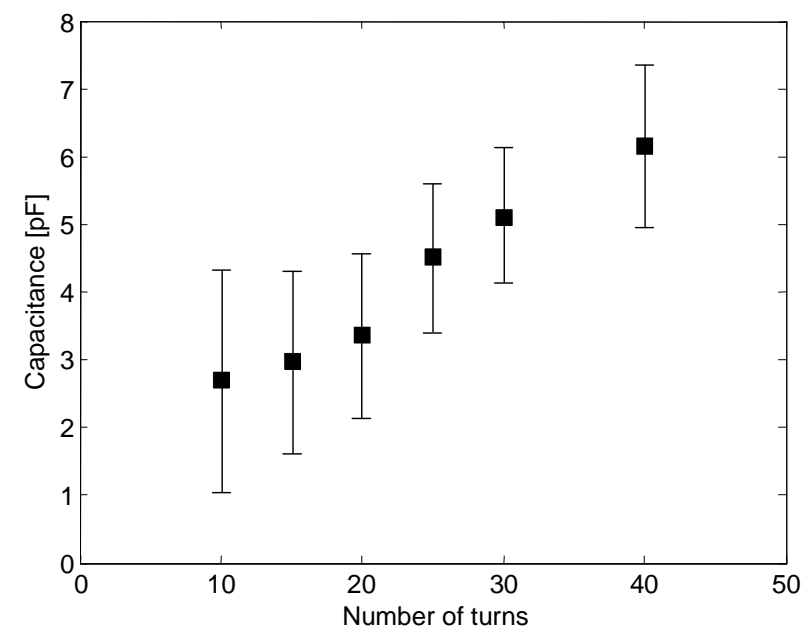

Fig. 6. Measured stray capacitance for the s.u.t. with $r=4 \mathrm{~mm}$ vs. number of turns $N$

The measurement results confirm an almost linear relationship of the capacitance value with respect to the number of turns.

\section{Uncertainty analysis}

In the following subsections the analysis of the uncertainty of the proposed method is subdivided in the evaluation of the uncertainty related to the adopted algorithm, that is a Least Mean Square regression that propagates the individual uncertainties of the input parameters to the uncertainty of the estimated $C_{p}$.

\subsection{Algorithm uncertainty}

When the stray capacitance $C_{p}$ is determined as the intercept of the linear regression operated on the measured pairs of values $\left(C_{e x t}, \lambda^{2}\right)$, the uncertainty is considered by defining the confidence intervals at $100 \times(1-\alpha) \%$ [13]. 
The expression (5) in reality identifies a linear relationship between the observed resonance frequency (in terms of $\lambda^{2}$, expressed as $l$ in the following by means of a change of variables) and the value of the external capacitor $C_{\text {ext }}$. The same expression written for the pairs $\left(C_{e x t, i}, l_{i}\right)$ of observed values takes the form

$$
C_{e x t, i}=\hat{A} \lambda_{i}^{2}-\hat{C}_{p}+e_{i}=\hat{A} l_{i}-\hat{C}_{p}+e_{i},
$$

where $e_{i}$ represents a sample of the error variable $e, \hat{A}$ and $\hat{C}_{p}$ indicate the estimations of the slope and intercept in the sense of the least mean square minimization [13].

It is known that the estimations of the slope $A$ and of the intercept $-C_{p}$ taken as linear combinations of the observed values, are unbiased estimators and thus bring to the "exact" values:

$$
E[\hat{A}]=A \quad E\left[\hat{C}_{p}\right]=C_{p} .
$$

An unbiased estimator of the variance of $e$ is

$$
\hat{\sigma}_{e}^{2}=\frac{\sum_{i=0}^{n-1} e_{i}^{2}}{n-2} .
$$

For the variance of the slope and of the intercept we may write

$$
\begin{gathered}
\operatorname{var}[\hat{A}]=\frac{\hat{\sigma}_{e}^{2}}{S_{l l}} ; \\
\operatorname{var}\left[\hat{C}_{p}\right]=\hat{\sigma}_{e}^{2}\left[\frac{1}{n}+\frac{\bar{l}^{2}}{S_{l l}}\right],
\end{gathered}
$$

where

$$
S_{l l}=\sum_{i=0}^{n-1}\left(l_{i}-\bar{l}\right)^{2}=\sum_{i=0}^{n-1} l_{i}^{2}-n \bar{l}^{2} \quad \text { with } \bar{l}=\frac{1}{n} \sum_{i=0}^{n-1} l_{i}
$$

The variance of the intercept identifies directly the uncertainty of the estimation of the stray capacitance $C_{p}$. While the first term between square brackets in (9) makes the variance decrease for an increasing number of observations $n$, the second term indicates that the distribution of the resonance frequency values is relevant to the reduction of the variance. In other terms, for the degenerate case of all the $l_{i}$ terms equal to a constant value $l^{*}$, the variance tends correctly to infinity. On the contrary, the wise distribution of the designed resonance frequency values at the two extremes of the frequency range, gets the minimum variance. Let's suppose that the set of $l_{i}$ values is subdivided into $n / 2$ points at $l_{d n}$ (the lower value) and $n / 2$ points at $l_{u p}$ (the upper value), with the two values symmetrical with respect to the central point, that represents the average value $\bar{l}$. While keeping constant the average value, the term $S_{l l}$ is made larger by increasing the number of points $n$ and by maximizing the distance of the two clusters $l_{u p}-l_{d n}=2 b$. The $l_{u p}$ and $l_{d n}$ values are symmetric around the average value $\bar{l}$, so that $S_{l l}=n b^{2}$; they correspond to the lower and higher nominal capacitance values, in the performed tests equal to $27 \mathrm{pF}$ and $2.2 \mathrm{nF}$ respectively. If $\bar{l} \cong b$, (10) simplifies to

$$
\operatorname{var}\left[\hat{C}_{p}\right] \cong \sigma_{e}^{2} \frac{2}{n} .
$$

The constraints for $l_{u p}$ and $l_{d n}$ are represented by the maximum measurable frequency of the frequency meter and by the extreme values of the external capacitors, that must be larger than the expected stray capacitance and smaller than a convenient maximum value, that ensures 
capacitance stability (with respect to temperature variations and ageing) by the availability of high quality dielectric materials (dielectric constant stability and low losses).

The confidence interval for the estimator of the intercept $\hat{C}_{p}$ is determined by observing that, if the observations are normally and independent distributed, the intercept is a $t$ random variable with $n-2$ degrees of freedom; thus, the amplitude of the confidence interval that brackets the true value $C_{p}$ at $100 \times(1-\alpha) \%$ probability is given by

$$
\hat{C}_{p}-t_{\alpha / 2, n-2} \sqrt{\sigma_{e}^{2} \frac{2}{n}} \leq C_{p} \leq \hat{C}_{p}+t_{\alpha / 2, n-2} \sqrt{\sigma_{e}^{2} \frac{2}{n}} .
$$

A $95 \%$ prediction interval corresponds to $\alpha=0.025$ and for $10 \leq n<\infty t_{\alpha / 2}=2.0 \pm 0.12$. The half-width of the confidence interval, that identifies the uncertainty related to the calculation method $u\left(C_{p}\right)$, is then approximately twice the dispersion indicated by (8).

\subsection{Instrumental and setup uncertainty}

Until now the values of the pair $\left(C_{e x t, i}, l_{i}\right)$ have been considered exact; yet, they are characterized by the instrumental uncertainty related to the determination of the values of the external capacitors and of the resonance frequency (that determines the parameter $l_{i}=\lambda_{i}^{2}$ ).

The external capacitors are high stability high quality capacitors, whose values were measured with a custom made capacitance bridge, featuring a very low uncertainty and high repeatability [14]; the maximum uncertainty for the lowest $C_{\text {ext }}$ values at $k=2$ coverage factor is lower than $100 \mathrm{ppm}$.

The instrumental uncertainty is minimized by the fact that the spectrum analyzer is used only to determine the resonance frequency $f_{r}$ and its accuracy (at $\left.k=2\right)$ is then better than $\pm(5$ $\mathrm{ppm}+1 \% \operatorname{span} / f_{r}+10 \% \mathrm{RBW} / f_{r}$, where span and RBW stand for frequency span and resolution bandwidth), as declared by the manufacturer with negligible aging [11]; with successive readings the frequency span and the resolution bandwidth may be reduced to conveniently low values, so that the total uncertainty never exceeds $100 \mathrm{ppm}$. The resonance frequency is identified by locating the peak in the $V_{r x}$ frequency response by reducing progressively the frequency span of the spectrum analyzer until the desired frequency resolution is obtained. It is observed that over a short frequency interval the top of the resonance peak is however difficult to locate, even if the vertical scale is adjusted accordingly; the reason is that small amount of noise, also picked up by the same RX coil, that produces clutter on the display: to this aim a suitable average option is advisable. The resonance frequency may be also determined by applying interpolation to the neighboring points once a given frequency span is reached.

The combined uncertainty for $u\left(C_{e x t}\right)$ and $u\left(f_{r}\right)$ at $k=2$ is thus lower than $140 \mathrm{ppm}$ and represents the most significant instrumental factor affecting the uncertainty on the $\left(C_{e x t, i}, l_{i}\right)$ pairs and thus the estimated variance of the error variable $e$. The setup uncertainty is approximately four to six larger.

\section{Conclusions}

The paper has considered the problem of determining the very low stray capacitance of single layer air solenoids with purposely separated turns. Measured values and expressions to be used as reference are very few in the literature, and they have been briefly reviewed to support the proposed measurement method. This method is based on linear regression applied to the pairs of values of the resonance frequency and the external resonating capacitance; the application of the driving signal and the measurement of the electrical variables is done 
without any galvanic connection to the solenoid under test, not to influence the stray capacitance terms, but the coupling is magnetic by means of an external launching coil and a current probe (or a receiving coil). The sources of uncertainty (both instrumental and related to confidence interval of the regression algorithm) have been considered and evaluated and the method has been applied to one test case with a solenoid made with $4 \mathrm{~mm}$ radius wire. The future development of this activity starts with the practical validation of the estimated confidence intervals of Section 4 with a Type A approach, repeating batch measurements on the same solenoid in different days and periods of the day to account for any external source of uncertainty not considered before. Moreover, also the repeatability and the influence of any test setup deviation will be considered by also modeling any non ideal characteristic of the test circuit and verifying its behavior and frequency response. Third, the availability of automatic or semi-automatic measurement operations is very important to reduce the measurement times. Finally, some reference solenoids will be constructed and shipped after measurement to other laboratories, in order to verify the accuracy of the method by direct comparison with other methods, such as high performance LCR bridges and Vector Network Analyzers. To this aim it is very important that any physical size limitation and connector specification are carefully discussed and agreed beforehand.

\section{Reference}

[1] Mariscotti, A. (18-23 May 2009). Rogowski winding with high voltage immunity. Proc. of EUROCON 2009. St. Petersburg. Russia. 1129-1133.

[2] Mariscotti, A., Vaccaro, L. (12-15 May 2008). A Rogowski coil for high voltage applications. Proc. of $I^{2}$ MTC 2008. Victoria. BC. Canada.

[3] Mariscotti, A. (2009). A Magnetic Field Probe with MHz Bandwidth and 7 decades Dynamic Range. IEEE Transactions on Instrumentation and Measurement. 58(8), 2643-2652.

[4] Mariscotti, A. (2009) Rail current measurement with non invasive large dynamic probe. IEEE Transactions on Instrumentation and Measurement. . 58(5), 1610-1616.

[5] Grandi, G., Kazimerzuc, M.K., Massarini, A., Reggiani, U. (1996). Stray capacitance of single-layer aircore inductors for high-frequency applications. IEEE Industry Application Conference. 3, 1384-1388.

[6] Medhurst, R.G. (1947). HF resistance and self capacitance of single layer solenoids - Part1. Wireless Engineering, 24, 35-43.

[7] Medhurst, R.G. (1947). HF resistance and self capacitance of single layer solenoids - Part2. Wireless Engineering. 24, 80-92.

[8] Rosa, E.B., Grover, F.W. (1948). Formulas and tables for the calculations of mutual and self inductance. Scientific papers of Bureau of Standards. Washington. 169.

[9] Mariscotti, A., Pozzobon, P. (2004). Resistance and Internal Inductance of traction rails: a survey. IEEE Transactions on Vehicular Technology. 53(4), 1069-1075.

[10] Mariscotti, A. (08-10 September 2010). On the measurement of the stray capacitance of single layer air solenoids. IMEKO TC4 Symposium. Kosice. SK.

[11] Agilent. (March 2007) N1996A Spectrum Analyzer - Specifications guide.

[12] Hewlett Packard. (1979). HP 5342A operating and service manual.

[13] Montgomery, D.C., Runger, G.C. (2003). Applied statistics and probability for engineers. John Wiley \& Sons. New York.

[14] Mariscotti, A. (2010). A low cost capacitive bridge based on voltage drop balance. Measurement. Elsevier. 43(9), 1094-1098. 